\title{
Déterminants Des Montants De Consentement À Payer (CAP) Déclarés Par Les Ménages Pour Une Gestion Durable De La Forêt d'Adjamey Au Sud- Ouest Du Bénin
}

\author{
Médard Miwoto \\ Afio Zannou \\ Gauthier Biaou
}

Laboratoire d'Economie Rurale et de Gestion des Exploitations Agricoles (LERGEA), Faculté des Sciences Agronomiques (FSA) de l'Université d’Abomey-Calavi (UAC), République du Bénin

doi: 10.19044/esj.2016.v13n3p293 URL:http://dx.doi.org/10.19044/esj.2016.v13n3p293

\begin{abstract}
The objective of this study is to analyze the factors that explain the amounts of willingness to pay (WTP) of households for the implementation of a sustainable management program of Adjamey forest in the district of Djakotomey in South-West of Benin. Indeed, the bordering populations of this forest are frequently faced with the problem of the devastation of their crops by the hippopotamuses. These in turn undergo anthropological actions causing the continued loss of their habitat. The data were collected on 110 bordering households using a semi-structured questionnaire. Based on the two-step selection model of Heckman, this study revealed that the households participation to the program of sustainable management of this forest is influenced by factors such as: the origin and level of education of the surveyed households, the fact of having been victims of the damage caused by the hippopotamuses and the fact that the household wishes an improvement of the management of this forest. Moreover, the study shows that the total area held in the forest, the total income drawn from the activities related to the forest, the membership in a management committee and the fact that the hippopotamuses are considered as a threat constitute to the explanatory factors of the amounts of willingness to pay (WTP) declared by the households.
\end{abstract}

Keywords: Willingness to pay, Adjamey forest, hippopotamuses, participation, Benin 


\section{Résumé}

L’objectif de cette étude est d'analyser les facteurs qui expliquent les montants de consentement à payer (CAP) des ménages pour la mise en œuvre d'un programme de gestion durable de la forêt d'Adjamey dans la Commune de Djakotomey au Sud-Ouest du Bénin. En effet, les populations riveraines de cette forêt sont fréquemment confrontées au problème de la dévastation de leurs champs de cultures par les hippopotames. Ces derniers subissent à leur tour les actions anthropiques entrainant la perte continue de leur habitat. Les données ont été collectées sur 110 ménages riverains à l'aide d'un questionnaire semi structuré. En se basant sur le modèle de sélection à deux étapes de Heckman, cette étude a révélé que la participation des ménages au programme de gestion durable de cette forêt est influencée par les facteurs tels que : l'origine et le niveau d'instruction de l'enquêté, le fait d'avoir subi les dégâts causés par les hippopotames et le fait que le ménage souhaite une amélioration de la gestion de la forêt. Aussi, a-t-elle montré que la superficie totale possédée dans le domaine forestier, le revenu total tiré des activités liées à la forêt, l’appartenance du ménage à un comité de gestion et la considération des hippopotames comme une menace constituent les facteurs explicatifs des montants de CAP déclarés par les ménages.

Mots-clés : Consentement à payer, forêt d'Adjamey, hippopotames, participation, Bénin

\section{Introduction}

La déforestation touche sévèrement les forêts tropicales. Elle entraîne des dommages économiques, sociaux et environnementaux auxquels il est difficile de remédier. De nombreuses études s’accordent à dire que les mauvaises politiques sont en général une des causes plus importante de la déforestation sous les tropiques (Folmer et al., 2007). Au Bénin, la problématique de la gestion des ressources naturelles se pose en terme de dégradation du couvert végétal, d’exploitation inadéquate des plans d'eau, de fortes pressions exercées sur les aires protégées (braconnage excessif, transhumance, activités agricoles, occupations anarchiques), d'appauvrissement quantitatif et qualitatif des terres cultivables (de Haan, 1992 ; Neef et Heidhues, 1993 ; Adégbidi et Biaou, 1994 ; Sounkéré, 2003 ; Houngnihin, 2005 ; Mèhou, 2007). De nos jours, le développement des cultures de rente a engendré une nouvelle vague de défrichement systématique au détriment des superficies qu'occupent les forêts et les réserves de faunes (Houngnihin, 2005; Mèhou, 2007). Or, les forêts constituent une source d'alimentation et d'énergie pour les millions d’habitants qui y vivent (Mèhou, 2007). 
La forêt d’Adjamey située dans la Commune de Djakotomey est victime d'une pareille dégradation de ses ressources naturelles. Cette forêt revêt une importance capitale en termes de conservation de la diversité biologique. La forte pression démographique, le caractère essentiellement agricole de l'activité économique et les pratiques agricoles extensives ont conduit à sa forte dégradation. La recherche d'espaces agricoles viables a amené les agriculteurs à occuper même le lit mineur des cours d'eau qui étaient déjà des zones naturelles de pâturage des hippopotames. Ces derniers, sont par ailleurs perturbés dans leur biotope par les pêcheurs et les éleveurs de bovins transhumants. Les affrontements hommes-hippopotames sont fréquents. Ainsi, la compétition pour la terre et l'eau a atteint des niveaux tels que la paix et la stabilité de la forêt sont menacées. D’une superficie estimée à 800 ha environ en 2010, cette forêt ne couvre qu'actuellement une superficie de 351 ha 97 ca (PIFSAP, 2013) soit une réduction de plus de la moitié (56\%) en trois (3) ans.

Par ailleurs, la dévastation des champs par les hippopotames est le problème récurrent que rencontrent les populations riveraines à la forêt d'Adjamey. Ces hippopotames subissent à leur tour la destruction continue de leur habitat. Compte tenu de l'importance socio-économique que revêt cette forêt telle refuge des espèces menacées (hippopotames), protection de la source d'eau 'Adjagan', barrière contre l'érosion des sols, potentiel d'écotourisme et valeurs spirituelles et religieuses, des programmes de préservation des ressources naturelles de la forêt doivent être mis en œuvre afin de contribuer à l'amélioration des conditions de vie des populations locales d'une part et de protéger la population animale en extinction d'autre part. De tels programmes ne peuvent avoir du succès sans le consentement et la participation effective des bénéficiaires. C'est dans ce contexte que les questions de recherches suivantes ont été formulées.

Quels sont les déterminants de la participation de ces bénéficiaires (populations riveraines) au programme de gestion durable? Quels peuvent être les facteurs explicatifs des montants de consentement à payer (CAP) déclarés par les ménages riverains?

Après une section exposant la méthodologie adoptée, une autre section exposera de façon détaillée les variables explicatives incluses dans le modèle de sélection à deux étapes de Heckman. La troisième section s'emploiera à exposer les résultats issus du logiciel STATA 13.0 sur les facteurs explicatifs des montants de CAP déclarés par les ménages. Enfin, une discussion des résultats saillants sera faite. 


\section{Méthodologie}

\section{Modélisation du consentement à payer (CAP)}

Le consentement à payer d'un ménage peut être estimé par la méthode dite d'évaluation contingente qui consiste à demander directement aux enquêtés la somme qu'ils sont prêts à payer pour un scénario fictif d'aménagement de l'environnement visant, par exemple, à empêcher sa détérioration ou à restaurer certaines parties endommagées.

Le modèle économétrique envisagé dans cette étude relève du domaine des modèles de sélection. En effet, la variable à expliquer est constituée des montants de CAP déclarés par les ménages, qui ne sont disponibles que pour ceux ayant accepté de participer financièrement au programme de gestion durable. Ce modèle, supposé séquentiel, est constitué de l'équation de sélection qui répond à la question "accepteriez-vous participer au programme de gestion durable de la forêt ? » et de l'équation substantielle répondant à la question "si oui à la question précédente, combien seriez-vous prêt à payer ? »

Il s'agit d'un modèle de sélection à deux étapes de Heckman. Dans un premier temps, le ménage choisit de participer au programme ou non, puis il décide du montant à payer. Bliki (2011) l'a utilisé pour étudier le financement de la gestion des déchets ménagers à Porto-Novo (Bénin). Il a été également utilisé par Danelutti (2003) pour une étude sur la consommation de bière, de vin et de spiritueux en Suisse. Deressa et al. (2010) l'ont aussi utilisé pour analyser les deux étapes du processus d'adaptation aux changements climatiques qui sont: 'perception du changement climatique par le producteur' et 'l'adaptation qu'il en fait'.

En se fondant sur le modèle développé par Heckman (1979), le modèle retenu pour la présente étude peut se formaliser comme suit pour chaque ménage $\mathrm{i}$.

\section{(1) Equation de sélection : Participation au programme de gestion durable de la forêt}

Soit $\mathrm{Z}$ la variable qualitative désignant la participation au programme, $Z=1$ si le ménage i participe au programme et 0 si non

$$
Z_{i}^{*}=W_{i} \gamma+\mu_{i}
$$

où on observe $\mathrm{Z}_{i}^{*}$ uniquement si l'individu $i$ accepte participer au programme.

$\mathrm{W}_{i}$ représente les variables socio-démographiques et économiques observables chez un ménage i, $\gamma$ est le paramètre estimé et $\mu_{i}$ est le résidu d'estimation qui suit une loi normale $N(0 ; 1)$.

(2) Equation substantielle : Estimation du CAP déclaré (observable uniquement si $Z=1$ ) 


$$
Y_{i}=X_{i} \beta+\varepsilon_{\mathrm{i}}
$$

avec $\mathrm{X}_{i}$ représentant les variables socio-démographiques et économiques observables chez un ménage $i, Y_{i}$ le montant de CAP du ménage $i, \beta$ est le paramètre estimé et $\varepsilon_{i}$ le résidu d'estimation qui suit une loi normale $N(0,1)$.

La Méthode du Maximum de Vraisemblance (MMV) permet d'estimer ce modèle. L'équation de participation est d'abord estimée par un modèle Probit; ensuite une régression par les Moindres Carrés Ordinaires (MCO) permet d'obtenir les coefficients de la deuxième équation. La variable à expliquer de l'équation de sélection a deux modalités (Oui =1 et Non $=0)$. Elle nécessite l'usage d'un modèle à choix binaire (McFadden et Léornard, 1993; Hanneman, 1996). La probabilité de répondre "Oui » correspond donc à la probabilité pour qu'une personne estime qu'elle est dans une meilleure situation avec le programme qui est proposé, même en contribuant financièrement. Le logiciel STATA 13.0 a été utilisé pour estimer le modèle de sélection à deux étapes de Heckman.

\section{Méthodes d'échantillonnage et de collecte des données}

L'étude s'est déroulée dans l'arrondissement de Kpoba, Commune de Djakotomey dans le département du Couffo au Sud-Bénin. Elle a porté sur les villages riverains de la forêt d'Adjamey (Figure 1). Quatre principaux critères tels que la position géographique du village par rapport à la forêt ; le poids démographique du village ; les groupes socio-culturels dominants et les activités dominantes ayant un impact sur la ressource ont facilité le choix des villages à enquêter. Au terme d'une enquête exploratoire, les villages Mèkpohoué et Fatouchèhoué ayant répondu à ces critères ont été retenus. Au total, 110 ménages sur une liste nominative de 260 ménages recensés ont été aléatoirement sélectionnés pour les enquêtes approfondies. Le guide d'entretien et le questionnaire sont les deux outils utilisés. 


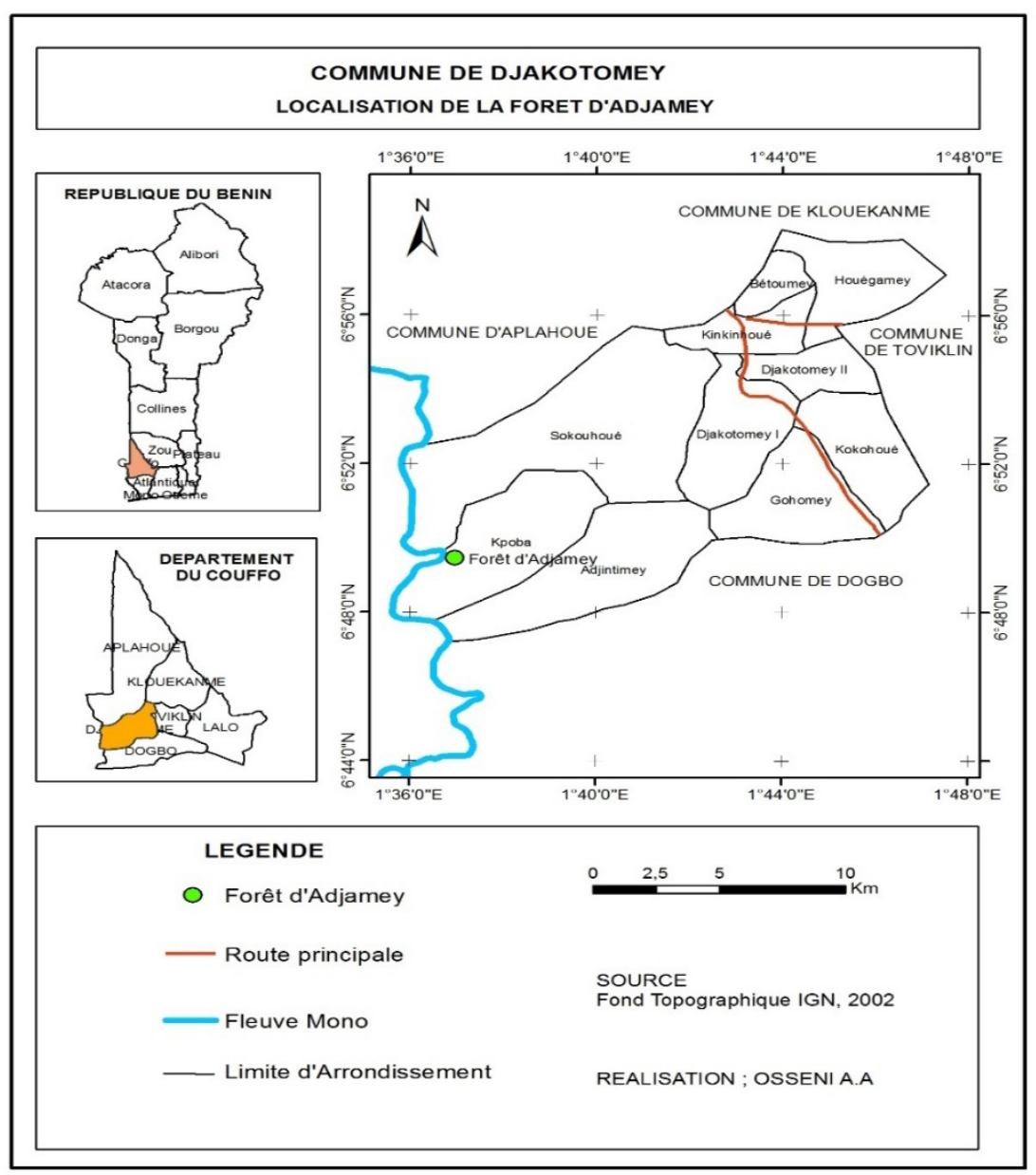

Figure 1 : Carte situant la forêt d’Adjamey au Bénin et dans la commune de Djakotomey

Variables explicatives incluses dans le modèle de sélection à deux étapes de Heckman

\section{- $\quad$ Equation de sélection}

Les variables expliquant la probabilité des ménages à participer au programme de gestion durable de la forêt sont les suivantes :

- AGE : est une variable continue mesurant l'âge du chef de ménage. Plus ce dernier est âgé, plus il a conscience des avantages liés à la préservation des ressources de la forêt. Une influence positive de cette variable est attendue.

- NINST: représente une variable binaire dénommée "'niveau d'instruction'. Un répondant, ayant été à l'école est plus disposé à participer à une gestion durable de la forêt. Un effet positif de cette variable est attendu. Un effet positif est donc attendu. 
- ORIG : désigne la variable binaire indiquant l'origine du chef de ménage (autochtone ou allochtone). Des études ont montré que les autochtones ont surtout tendance à adopter les innovations (Nkamleu and Adesina, 1998). La variable origine influence alors positivement la participation du ménage au programme.

- SOUHAIT : Elle désigne le fait que les ménages souhaitent ou non l'amélioration de la gestion de la forêt. Les ménages qui souhaitent l'amélioration de la gestion de la forêt sont plus disposés à participer au programme. Un effet positif est donc attendu.

- SUBDHIP : est la variable binaire traduisant le fait que le ménage ait une fois ou pas subi les dégâts causés par les hippopotames. Les ménages ayant subi les affres de ces derniers acceptent participer au programme de gestion durable de la forêt. Un effet positif est donc espéré.

\section{- $\quad$ Equation substantielle}

Les variables explicatives du montant de CAP déclaré par les ménages riverains de cette forêt sont :

- MENACE : représente la variable binaire traduisant la considération des hippopotames comme une menace ou non par le ménage. Un ménage, pour qui ces animaux constituent une menace, va déclarer un montant élevé pour contribuer à la gestion durable de la forêt. Un signe positif du coefficient associé à cette variable est attendu.

- SUP : représente la superficie totale possédée par le chef ménage dans la forêt. Elle a été prise en compte afin d'apprécier son effet sur le montant que les ménages riverains ont déclaré comme participation à la gestion durable de la forêt. Plus la superficie totale possédée est élevée, plus le ménage est prêt à participer financièrement au programme. Une influence positive de cette variable sur les montants déclarés est alors attendue.

- REVTIRE : est une variable continue qui mesure le revenu total que le ménage tire des activités liées à la forêt. Plus un ménage tire un revenu élevé, plus il déclare un montant élevé comme contribution financière à la gestion durable de la forêt. Un signe positif est alors attendu du coefficient associé à cette variable.

- MACGEST : représente ''l'appartenance au comité local de gestion de l'Association Villageoise de Gestion des Réserves de Faune (AVIGREF) ou du comité des Ecogardes'. Elle est une variable binaire qui prend la valeur 1 si le chef de ménage est/ou a été membre de l'AVIGREF ou du comité des écogardes et 0 si non. Les membres des comités locaux de gestion sont prêts à beaucoup contribuer à la gestion durable de la forêt. Un effet positif est attendu de cette variable.

Le type, les niveaux de mesure et les signes attendus de ces différentes variables sont consignés dans le tableau 1. 
Tableau 1 : Définitions des variables explicatives

\begin{tabular}{|c|c|c|c|c|}
\hline Variables & Désignation & Type & Niveau de mesure & $\begin{array}{l}\text { Signes } \\
\text { attendus }\end{array}$ \\
\hline $\begin{array}{c}\text { Equation de sélection } \\
\text { Acceptation de participer au } \\
\text { programme }\end{array}$ & ACCEPT & Binaire & $1=$ Oui ; $0=$ Non & \\
\hline Age de l'enquêté & AGE & Continue & Années & + \\
\hline Niveau d’instruction du répondant & NINST & Binaire & $1=$ Instruit ; $0=$ Si non & + \\
\hline Origine de l'enquêté & ORIG & Binaire & $\begin{array}{c}\text { 1=Autochtone; } 0= \\
\text { Allochtone }\end{array}$ & + \\
\hline $\begin{array}{l}\text { Souhait de l'amélioration de la gestion } \\
\text { du site }\end{array}$ & SOUHAIT & Binaire & $\begin{array}{l}1=\text { Souhaite } \\
\text { amélioration } ; 0=\text { Si non }\end{array}$ & + \\
\hline $\begin{array}{c}\text { Avoir subi les dégâts des } \\
\text { hippopotames } \\
\text { Equation substantielle }\end{array}$ & SUBDHIP & Binaire & $1=$ Oui ; $0=$ Non & + \\
\hline Montant déclaré par le ménage & MNTPAY & $\begin{array}{c}\text { Continu } \\
\mathrm{e}\end{array}$ & Montant en FCFA & \\
\hline $\begin{array}{c}\text { Considère les hippopotames comme } \\
\text { une menace }\end{array}$ & MENACE & Binaire & $1=$ Oui ; $0=$ Non & + \\
\hline Superficie totale possédée & SUP & $\begin{array}{c}\text { Continu } \\
\text { e }\end{array}$ & Valeur en hectare & + \\
\hline Revenu total tiré des activités menées & REVTIRE & $\begin{array}{c}\text { Continu } \\
\mathrm{e}\end{array}$ & Valeur en FCFA & + \\
\hline Membres des comités de gestion & MACGEST & Binaire & $1=$ Oui ; $0=$ Non & + \\
\hline
\end{tabular}

\section{Résultats et discussion}

Statistiques descriptives des variables incluses dans le modèle

Le tableau 2 présente les statistiques descriptives de toutes les variables explicatives incluses dans l'équation de sélection et dans l'équation substantielle.

Tableau 2 : Statistiques descriptives des variables explicatives incluses dans le modèle de Heckman

\begin{tabular}{ccc}
\hline Variables explicatives incluses dans le modèle de & Moyenne & Ecart-type \\
Heckman & & \\
\hline Equation de sélection & & 10,164 \\
Age de l'enquêté & 46,036 & 0,060 \\
Niveau d'instruction du répondant & 0,836 & 0,018 \\
Origine de l'enquêté & 0,963 & 0,048 \\
Souhait de l'amélioration de la gestion & 0,491 & 0,039 \\
Fait d'avoir subi les dégâts des hippopotames & 0,791 & 0,044 \\
Pratique de la chasse dans la forêt & 0,709 & \\
Equation de substantielle & & 0,401 \\
Considération des hippopotames comme une menace & 0,800 & 2,003 \\
Superficie possédée & 1,955 & 24630,33 \\
Revenu total tiré des activités liées à la forêt & 236497,7 & 0,046 \\
Appartenance à un comité de gestion & 0,409 & \\
\hline
\end{tabular}




\section{Résultats de l'estimation économétrique}

A partir du modèle de sélection à deux étapes de Heckman, les coefficients de l'équation de sélection s’interprètent comme ceux du modèle Probit. Quant aux coefficients de l'équation substantielle, ils représentent l'influence de la variable explicative sur la variable expliquée. Il s’agit d'une Méthode des Moindres Carrés Ordinaires.

Les résultats (tableau 3) indiquent que l'estimation de l'équation de sélection a porté sur la totalité des individus ayant répondu au scénario contingent c'est-à-dire les 110 ménages enquêtés. Les observations non censurées c'est-à-dire les observations des individus ayant décidé de payer sont considérées dans l'équation substantielle. Cette estimation a porté sur 78 observations non censurées. Par ailleurs, la significativité de la fonction de probabilité du modèle de sélection de Heckman (Wald $\chi^{2}(5)=60,52$; Prob $\left.>\chi^{2}=0,0001\right)$ montre la forte puissance explicative de ce modèle. De plus, le coefficient du ratio de Mills est significatif à 5\% (P=0,020<5\%) et par conséquent, le modèle est globalement significatif au seuil de $5 \%$. Il s'en suit que la décision de révéler le montant à payer n'est pas prise indépendamment de la décision d'être disposé à participer au programme de gestion durable de la forêt d'Adjamey. Aussi, ces résultats permettent-ils d'affirmer que le modèle de sélection de Heckman est mieux adapté aux observations. Ainsi, les ménages riverains de la forêt d'Adjamey accordent une importance à l'amélioration de sa gestion et sont prêts à y contribuer.

Tableau 3 : Résultat de l'estimation à deux étapes de Heckman de l'équation de sélection et de l'équation substantielle.

\begin{tabular}{|c|c|c|c|}
\hline Variables & Coefficient. & $\begin{array}{l}\text { Erreur } \\
\text { standard }\end{array}$ & $\mathrm{P}>\mathrm{Z}$ \\
\hline \multicolumn{4}{|l|}{ Equation de sélection } \\
\hline Age de l'enquêté & 0,001 & 0,001 & 0,350 \\
\hline Niveau d'instruction du répondant & 0,182 & 0,025 & $0,000 * * *$ \\
\hline Origine de l'enquêté & $-0,182$ & 0,060 & $0,002 * * *$ \\
\hline Souhait de l'amélioration de la gestion & $-0,037$ & 0,021 & $0,072 *$ \\
\hline Fait d'avoir subi les dégâts des hippopotames & 0,051 & 0,025 & $0,049 * *$ \\
\hline Constante & 1,460 & 0,104 & $0,000 * * *$ \\
\hline \multicolumn{4}{|l|}{ Equation substantielle } \\
\hline Superficie possédée & $-0,481$ & 0,143 & $0,001 * * *$ \\
\hline Revenu total tiré des activités liées à la forêt & $1,55 \mathrm{e}^{-06}$ & $8,67 \mathrm{e}^{-07}$ & $0,074^{*}$ \\
\hline Considération des hippopotames comme une menace & 3,910 & 0,924 & $0,000 * * *$ \\
\hline Appartenance à un comité de gestion & 2,334 & 0,633 & $0,000 * * *$ \\
\hline Constante & $-2,965$ & 0,815 & $0,000 * * *$ \\
\hline Mills lambda & 0,083 & \multicolumn{2}{|c|}{$0,036 \quad 0,020^{* *}$} \\
\hline Rho & \multirow{2}{*}{\multicolumn{3}{|c|}{$\begin{array}{l}0,909 \\
0,092\end{array}$}} \\
\hline Sigma & & & \\
\hline \multicolumn{4}{|c|}{ Nombre d'observations $=110 ;$ Observations non censurées $=78 ;$ Observations censurées $=32 ;$} \\
\hline Prob $>\chi^{2}=0,0001 ;$ & ${ }_{\mathrm{d}} \chi^{2}(5)=$ & $60,52$. & \\
\hline
\end{tabular}


Facteurs influençant la participation (CAP) des ménages au programme

Le coefficient de la variable ''niveau d'instruction'” introduite dans le modèle de sélection de Heckman est significative au seuil de $1 \%$ et montre que cette variable influence positivement la participation des enquêtés. La banque mondiale (1993), Vasquez (2009) et Gbinlo (2010) ont également trouvé que le niveau d'éducation influence positivement le CAP des ménages. Ainsi, lorsque le répondant a été à l'école, il est plus disposé à participer à la gestion de la forêt. Cette situation s'explique par le fait que les enseignements théoriques que ces ménages ont reçus les prédisposent à cerner l'importance de la gestion durable des ressources naturelles en général et celle de la forêt d'Adjamey en particulier.

La variable 'origine du chef ménage'” a une influence négative sur la décision des ménages à participer financièrement au programme de gestion durable de la forêt. Ce résultat est contraire aux propos de la plupart des autochtones qui ont compris l'importance de sa conservation surtout de la protection des animaux en voie de disparition comme les hippopotames. Ceci se justifie par les différentes séances de sensibilisation sur les services écosystémiques de cette forêt et la visite de certains sites régionaux de conservation des hippopotames qui ont amené ces ménages à adopter des comportements éco-citoyens. Pour eux, cette gestion durable offrira une meilleure visibilité et permettra à leur arrondissement d'accéder aux infrastructures socio-communautaires.

'Le fait d'avoir une fois subi les dégâts des hippopotames" est une variable qui s'est révélée significative avec un signe positif comme prévu. Cela signifie que la probabilité de participer au programme de gestion de la forêt augmente avec le fait que le ménage ait une fois subi les dégâts des hippopotames. En effet, compte tenu de la satisfaction obtenue après la gestion qui avait été faite, les ménages accordent une importance à une gestion plus rationnelle et durable de cette forêt. Ils sont, par conséquent, disposés à contribuer financièrement à cette gestion car il y va de leur intérêt.

La variable 'souhait de l'amélioration de la gestion de la forêt' est significativement différent de zéro. La question de la gestion durable des ressources est donc essentielle pour les ménages riverains de la forêt d’Adjamey. Ceux qui souhaitent une amélioration de la gestion actuelle doivent plus accepter participer au programme. Or, avec ce modèle, cette variable à une influence négative sur l'acceptation du ménage à participer à la gestion durable de cette forêt. Cela s'explique par le fait que, malgré qu'ils souhaitent l'amélioration de la gestion de cette forêt, ils n'acceptent pas contribuer financièrement au programme puisse que selon eux, toute participation financière incombe aux institutions chargées de la gestion des ressources naturelles. 


\section{Facteurs influençant les montants de CAP déclarés par les ménages}

'La superficie de terre possédée par les ménages dans le domaine forestier' est déterminante dans la proposition du montant que le ménage accepte payer. Elle a une influence négative sur ce montant. Ainsi, plus les ménages disposent d'une grande superficie, plus ils déclarent de faibles montants en guise de participation à la gestion durable de la forêt d'Adjamey. Cette situation montre que les ménages détenteurs de grandes superficies ressentent moins les affres des hippopotames.

''La considération des hippopotames comme une menace par les ménages"' a une influence positive sur les montants que ces ménages ont déclarés pour contribuer à la gestion durable de la forêt. Cette variable est significative au seuil de 1\%. Ainsi, plus cette menace est considérée comme grande par un ménage, plus élevée est sa contribution. En effet, 80\% des enquêtés considèrent les hippopotames comme une menace puisqu'ils subissent fréquemment la dévastation de leurs champs par ces hippopotames. Ces animaux détruisent en un temps record d'énormes emblavures sur leur trajectoire. Cette situation a pour corollaire d'importantes pertes de récoltes, la baisse du revenu des ménages et l'insécurité alimentaire. Ce résultat est conforme à celui de Bliki (2011) qui stipule que les ménages qui considèrent les ordures ménagères comme une menace pour la santé révèlent un montant de CAP élevé.

La variable "'revenu total tiré des activités liées à la forêt'” a une influence positive et significative sur le montant de CAP proposé par les ménages comme participation financière au programme de gestion durable de la forêt. L’effet estimé correspond à l'effet attendu. Ce résultat est conforme à ceux de Casey et al. (2006) et de Bliki (2011) selon lesquels le revenu est positivement lié au CAP. Ainsi, plus un ménage tire un revenu élevé des activités liées à la forêt, plus il déclare un montant élevé. Ceci se traduit par le fait que les ménages tirant un revenu élevé espèrent en avoir davantage à travers leur participation financière à la mise en œuvre effective du programme de gestion durable de la forêt.

Des résultats issus du modèle, il ressort que la variable ', l'appartenance du chef ménage à un comité local de gestion de la forêt', est significative à $1 \%$ et a une influence positive sur le montant que le ménage est disposé à payer. Ce qui est conforme au signe prédit. Ainsi, les ménages membres qui sont/ou ayant été membres des comités locaux de gestion sont plus disposés à payer des montants élevés pour une gestion durable de la forêt d’Adjamey. En effet, les ménages ayant participé aux diverses activités des comités locaux de gestion ont une bonne connaissance des réelles difficultés notamment financières et sont par conséquent plus disposés à déclarer des montants élevés pour contribuer au programme de gestion durable de la forêt. 


\section{Conclusion}

Il ressort de l'étude que la participation des ménages au programme de gestion durable de la forêt d'Adjamey est influencée par les facteurs tels que : l'origine et le niveau d'instruction de l'enquêté, le fait d'avoir subi les dégâts causés par les hippopotames et le fait que le ménage souhaite une amélioration de la gestion de la forêt. Les coefficients des quatre variables qui déterminent les montants de CAP que les ménages sont disposés à payer sont significativement différents de zéro. En définitive, les montants déclarés par les ménages pour participer à la gestion durable de la forêt d'Adjamey sont influencés par la superficie totale possédée dans le domaine forestier, le revenu total tiré des activités liées à la forêt, l’appartenance du ménage à un comité de gestion et la considération des hippopotames comme une menace.

Pour les décideurs publics, les Partenaires Techniques et Financiers ayant en charge la gestion des forêts au Bénin en général et dans la Commune de Djakotomey en particulier, ces résultats peuvent les éclairer sur les réelles motivations des ménages face à la mise en œuvre des projets et programmes de gestion durable des ressources naturelles qui les responsabilisent.

\section{References:}

1. Adégbidi, A., Biaou, G., 1994. L’agriculture durable au Bénin : rôle de l'état. In : de Haan et P. Ton (éds) (1995). A la recherche de l’agriculture durable au Bénin. UVA, Amsterdam 1995.

2. Bliki, S. A., 2011. Financement de la gestion des déchets ménagers à Porto-Novo au Bénin. Mémoire de fin de formation pour l'obtention du DEA/Master. UAC

3. Casey, J. F., Kahn, J. R., Rivas, A., 2006. Analysis willingness to pay for improved water service in Manaus, Amazonas, Brazil. Ecological Economics 58 (2006) 365-372.

4. Danelutti, T., 2003. Une étude sur la consommation de bière, de vin et de spiritueux en suisse. Statistiques et économétrie appliquées 2. Ecole des HEC - Université de Lausanne. 36 p.

5. de Haan, L., 1992. Rapport entre agriculteurs et éleveurs au Nord Bénin : écologie et interdépendance transformée. Tome 1\&2 Amsterdam, 1992.

6. Deressa, T. T., Hassan, R. M., Ringler, C., 2010. Perception of and adaptation to climate change by farmers in the Nile basin of Ethiopia. Journal of agricultural science (2011), 149, 23-31.

7. Folmer, H., Van Kooten, G., 2007. Deforestation dans Solutions for the world's biggest problems: costs and benefits. Lomborg (Sous la direction de), Cambridge, Royaume-Uni, Cambridge University Press. 
8. Gbinlo, E. R., 2010. Organisation et financement de la gestion des déchets ménagers dans les villes de l'Afrique Subsaharienne : le cas de la ville de Cotonou au Bénin. Economies et finances. Thèse de doctorat en Sciences Economiques. Université d’Orléans. pp 110-141

9. Hanemann, W. M., 1978. A methodological and empirical study of the recreation benefits from water quality improvement, Cambridge, MA, Harvard University.

10. Heckman, J., 1979. Sample Selection Bias as a Specification Error. Econometrica, 47(1): 153-161.

11. Houngnihin, R., 2005. Les mécanismes endogènes dans la problématique de l'environnement à Covè. $11^{\text {ème }}$ assemblée générale du CODESRIA à Mapouto du 06 au 10 décembre 2005.

12. Maler, K. G., 1974. Environmental Economics: A Theoretical Inquiry John Hopkins University Press, Baltimore, MD.

13. McFadden, D., 1994. Contingent valuation and social choice, American Journal of Agricultural Economics, 76, p. 689-708.

14. Mèhou, S. R-B., 2007. Gestion foncière dans les terroirs riverains de la réserve de biosphère de la Pendjari. Thèse d'Ingénieur Agronome FSA/UAC.

15. Neef, A., Heidhues F., 1993. The role of land tenure in agroforestry: lessons from Benin. Agroforestry Systems (27).

16. Nkanleu, N.G.B., Adesina, A. A., 1998. Multinominal logit analysis of soil nutrient management technologies in Cameroon. Agricultural economics (for submission).

17. PIFSAP, 2013. Plan d'aménagement et de gestion simplifie de la forêt sacrée Adjamey. ' Site aux hippopotames', 52 p.

18. Sounkéré, K., 2003. Analyse des formes d’utilisation de l'espace dans l’Atacora. Mémoire pour l’obtention du diplôme d’ingénieur agronome. FSA/UAC. Bénin.

19. Vasquez, W. F., Mozumder, P., Hernandez-Arce, J., Berrens, R. P., 2009. Willingness to pay for safe drinking water: Evidence from Parral, Mexico. Journal of Environmental Management 90. pp. 33913400 .

20. World Bank, 1993. The demand for water in rural areas: determinants and policy implications. The World Bank Research Observer. vol.8, no.1, January 1993. pp. 47-70. 